1. Tobler, I. 2005. Phylogeny of sleep regulation. In Principles and practice of sleep medicine. M.H. Kryger, T. Roth, and W.C. Dement, editors. 4th edition. W.B. Saunders. Philadelphia, Pennsylvania, USA. 77-90.

2. Durmer, J.S., and Dinges, D.F. 2005. Neurocognitive consequences of sleep deprivation. Semin. New rol. 25:117-129.

3. Jones, B.E. 2005. From waking to sleeping: neuronal and chemical substrates. Trends Pharmacol. Sci. 26:578-586

4. Dahan, L., et al. 2007. Prominent burst firing of dopaminergic neurons in the ventral tegmental area during paradoxical sleep. Neuropsychopharmacology. 32:1232-1241.

5. Monti, J.M., and Monti, D. 2007. The involvement of dopamine in the modulation of sleep and waking. Sleep Med. Rev. 11:113-133.

6. Sakurai, T. 2007. The neural circuit of orexin (hypocretin): maintaining sleep and wakefulness. Nat. Rev. Neurosci. 8:171-181.

7. Denoyer, M., Sallanon, M., Buda, C., Kitahama, K., and Jouvet, M. 1991. Neurotoxic lesion of the mesencephalic reticular formation and/or the posterior hypothalamus does not alter waking in the cat. Brain Res. 539:287-303.

8. McCormick, D.A., and Bal, T. 1997. Sleep and arousal: thalamocortical mechanisms. Ann. Rev. Neurosci. 20:185-215.

9. Sherin, J.E., Shiromani, P.J., McCarley, R.W., and Saper, C.B. 1996. Activation of ventrolateral preoptic neurons during sleep. Science. 271:216-219.

10. Suntsova, N., Szymusiak, R., Alam, M.N., Guzman-Marin, R., and McGinty, D. 2002. Sleep-waking discharge patterns of median preoptic nucleus neurons in rats. J. Physiol. 543:665-677.

11. Detari, L., Rasmusson, D.D., and Semba, K. 1997. Phasic relationship between the activity of basal forebrain neurons and cortical EEG in urethaneanesthetized rat. Brain Res. 759:112-121.

12. Lee, M.G., Manns, I.D., Alonso, A., and Jones, B.E. 2004. Sleep-wake related discharge properties of basal forebrain neurons recorded with micropipettes in head-fixed rats. J. Neurophysiol. 92:1182-1198.

13. Borbély, A.A. 1982. A two process model of sleep regulation. Human Neurobiol. 1:195-204.

14. Rao, Y., et al. 2007. Prolonged wakefulness induces experience-dependent synaptic plasticity in mouse hypocretin/orexin neurons. J. Clin. Invest.
117:4022-4033.

15. McDermott, C.M., et al. 2003. Sleep deprivation causes behavioral, synaptic, and membrane excitability alterations in hippocampal neurons. J. Neurosci. 23:9687-9695.

16. Kopp, C., Longordo, F., Nicholson, J.R., and Luthi, A. 2006. Insufficient sleep reversibly alters bidirectional synaptic plasticity and NMDA receptor function. J. Neurosci. 26:12456-12465.

17. Tartar, J.L., et al. 2006. Hippocampal synaptic plasticity and spatial learning are impaired in a rat model of sleep fragmentation. Eur. J. Neurosci. 23:2739-2748.

18. Rioult-Pedotti, M.S., Friedman, D., and Donoghue, J.P. 2000. Learning-induced LTP in neocortex. Science. 290:533-536.

19. Whitlock, J.R., Heynen, A.J., Shuler, M.G., and Bear, M.F. 2006. Learning induces long-term potentiation in the hippocampus. Science. 313:1093-1097.

20. Tononi, G., and Cirelli, C. 2006. Sleep function and synaptic homeostasis. Sleep Med. Rev. 10:49-62.

21. Kilduff, T.S., and Peyron, C. 2000. The hypocretin/ orexin ligand-receptor system: implications for sleep and sleep disorders. Trends Neurosci. 23:359-365.

\title{
Structural reengineering of imatinib to decrease cardiac risk in cancer therapy
}

\author{
George D. Demetri
}

Ludwig Center for Cancer Research, Dana-Farber Cancer Institute and Harvard Medical School, Boston, Massachusetts, USA. Clinical and Translational Research, Ludwig Institute for Cancer Research, New York, New York, USA, and Zurich, Switzerland.

\begin{abstract}
Imatinib, a selective, small-molecule tyrosine kinase inhibitor, has life-saving clinical activity in certain cancers, but questions have been raised about the potential for cardiac toxicity through inhibition of its target, ABL kinase. In this issue of the JCI, Fernández et al. describe a novel method by which the ABL-inhibitory activity of imatinib was deleted by modifying its chemical structure (see the related article beginning on page 4044). The anticancer activity of the reengineered agent, called $\mathrm{WBZ}_{-} 4$, was instead preserved against gastrointestinal stromal tumors in both in vitro and in vivo models via inhibition of KIT tyrosine kinase, and the desired safety was demonstrated with less cardiotoxicity of $\mathrm{WBZ}_{4} 4$ compared with imatinib via the inhibition of JNK. The study shows that structural reengineering of a kinaseinhibitory drug to improve tolerability while preserving efficacy is feasible.
\end{abstract}

\section{Signal transduction inhibitors in cancer therapy}

The molecular genetic basis for many forms of cancer has been elucidated; the etiologic connection between mutational activation of certain oncogenic gene products and

Nonstandard abbreviations used: CML, chronic myelogenous leukemia; GIST, gastrointestinal stromal tumor.

Conflict of interest: The author receives research support from Ariad Pharmaceuticals Inc., Bristol-Myers Squibb Co., Daiichi Sankyo Inc., Infinity Pharmaceuticals, Johnson \& Johnson, Novartis Pharmaceuticals Corp., and Pfizer Inc. and consultant fees from Ariad Pharmaceuticals Inc. and Ziopharm Oncology Inc.

Citation for this article: J. Clin. Invest. 117:3650-3653 (2007). doi:10.1172/JCI34252. human cancer are now fully evident for certain diseases such as chronic myelogenous leukemia (CML) and gastrointestinal stromal tumors (GISTs), in which single mutations lead to uncontrolled tyrosine kinase activity of the BCR-ABL fusion kinase and the KIT kinase, respectively. The concept of using rationally designed small molecules to inhibit such oncogenic kinase signaling to treat cancer had its roots in the work of Alexander Levitzki and others in the 1990s (1). However, the successful implementation of this concept came with the dramatic success of imatinib mesylate (now known as Gleevec in the United States and Glivec in the rest of the world), which has revolutionized the treatment of patients with CML. A multi-institutional collaboration between Brian Druker and Nicolas Lyden and their colleagues with pharma led to the focused testing of imatinib to target BCR-ABL kinase activity $(2,3)$, and subsequent multi-institutional collaborations targeted the KIT kinase in GISTs as another therapeutic application $(2,4)$. The result of this work has given hope to the field of oncology, validating the concept that both hematologic malignancies and solid tumors in humans can be treated successfully with agents that target elements of the molecular pathogenesis of the disease. Both CML and GISTs were ideal proof-of-concept diseases, because single genetic mutations play a disproportionately large role in the pathobiology of these diseases.

\section{Clinical impact of selective or multitargeted kinase inhibitors in cancer treatment}

Imatinib has become the "poster child" drug of small-molecule molecular therapeutics, with a spectrum of activity that is reasonably selective for certain cell signaling pathways. It is important to note that 
A

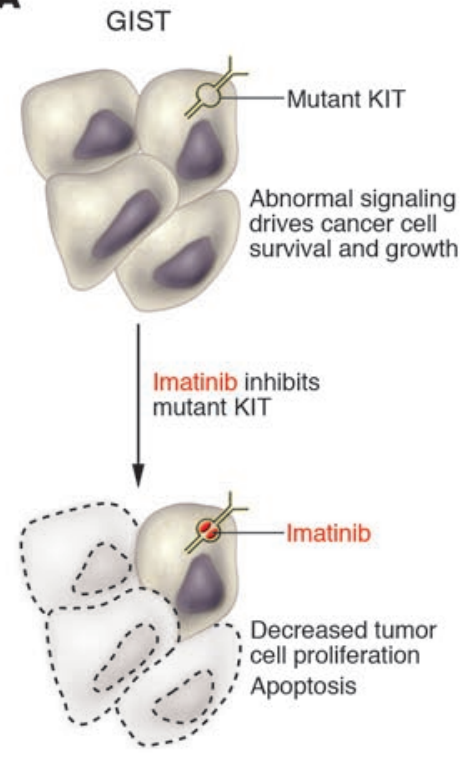

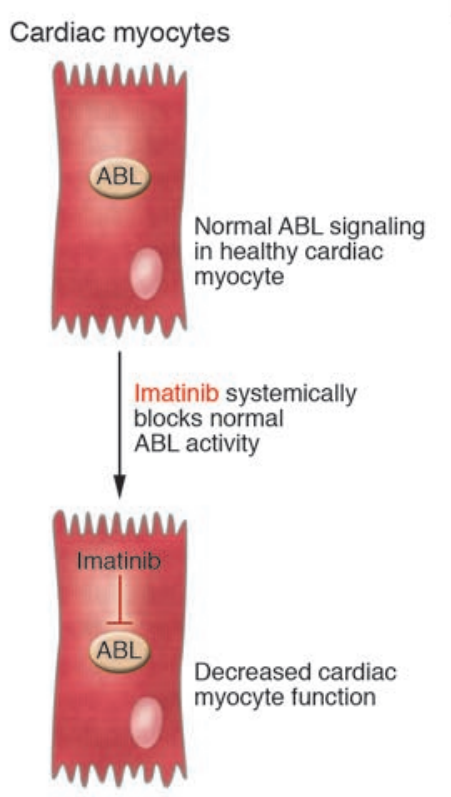

B
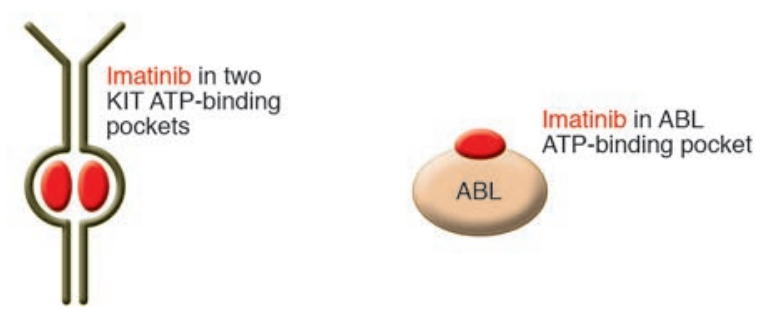

De-wetting profiles examined, new ligand designed (WBZ_4)

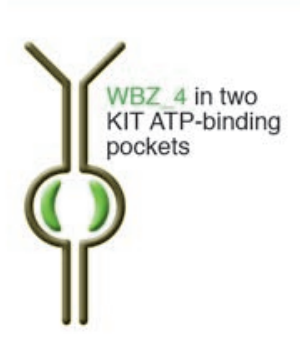

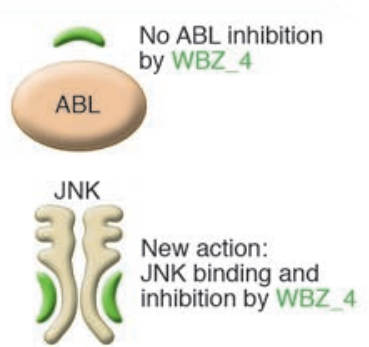

Figure 1

Structural modification of imatinib allows retention of its anticancer activity without cardiotoxic effects. (A) Imatinib is able to bind to the ATPbinding pocket of tyrosine kinases and therefore block the mutant KIT receptor tyrosine kinase activity in GIST cells, leading to inhibition of intracellular signaling, decreased cell proliferation, and apoptosis of the cancer cells. Cardiac myocytes exhibit normal signaling through the intracellular ABL kinase. Imatinib-mediated inhibition of the ABL kinase results in decreased cardiac myocyte function. (B) Imatinib serves as a ligand that binds to the ATP-binding pocket of KIT as well as ABL kinases. In this issue of the $J C l$, Fernández et al. (13) examined the de-wetting profiles of KIT and BCR-ABL and reengineered imatinib to create WBZ_4. This compound was designed to retain binding to KIT ATP-binding pockets, not to bind ABL, and to bind to and inhibit JNK kinase for the purpose of increased cardioprotection.

no small molecule can possibly provide complete specificity, as might be obtained from a monoclonal antibody, for example. Although relatively selective, imatinib inhibits a number of different tyrosine kinases, including the ABL family (encompassing the oncogenic fusion protein product of the leukemogenic BCR-ABL translocation as well as ABL and the ABL-related gene ARG), as well as having potent activity against mutated oncogenic forms of the receptor tyrosine kinases KIT and the platelet-derived PDGFR- $\alpha$ (2). The clinical benefits of this selective tyrosine kinase inhibitor are dramatic, durable, and impressively well tolerated for the majority of patients with CML (3) and GISTs (4, 5). Imatinib has more than tripled overall survival for GIST patients with advanced metastatic disease, although the clonal evolution of genotypically distinct GIST cells that are resistant to the kinase-inhibitory effects of imatinib limits the long-term benefits for the majority of patients $(6,7)$. Other kinase inhibitors with an even broader spectrum of activity (so-called "multitargeted" inhibitors) have shown benefit for patients following failure of imatinib, including sunitinib for GISTs (6) and dasatinib for CML (8).

\section{Risks of kinase inhibition in normal cardiac myocytes}

Much attention has recently surrounded the potential risk of tyrosine kinase inhibitors to induce some measure of cardiac dysfunction. While conflicting data have been presented, and while the clinical conclusions remain somewhat controversial, the laboratory-based work that forms the foundation of this concern is compelling. Inhibition of $\mathrm{ABL}$ in normal cardiac myocytes appears to impair their well-being and could lead to functional damage in patients $(9,10)$. The initial report of this concern for imatinib was limited by a certain selection bias: while 10 cases of cardiac dysfunction were reported in patients treated with imatinib, there was no mention of the overall size of the underlying study population exposed to imatinib (so that incidence and risk statistics could be assessed), nor was there mention of known prior cardiac risk factors in these initial 10 patients (9). Many anticancer agents such as anthracycline chemotherapy agents have known cardiac risks; other physiologic risks, such as the increased total body fluid load and edema that are known to be associated with imatinib therapy, were not defined separately. Subsequent studies have noted the very low incidence of significant cardiotoxicity with imatinib $(11,12)$; other data are emerging about the potential of other multitargeted tyrosine kinase inhibitors to have negative cardiovascular effects (10). Putting the benefits of these agents in perspective requires further understanding of individual risk factors and of the true key physiologic signaling mechanisms in cardiac myocytes affected by these inhibitors, which may differ from signaling pathways active in cancer cells. A key clinical question remains: Is the beneficial impact of imatinib or other tyrosine kinase inhibitors truly limited in a meaningful way by cardiotoxicity, or is this a minor issue that pales in comparison to the more pressing problem of tumor cell resistance to any small-molecule tyrosine kinase inhibitor?

\section{Rational reengineering of a targeted anticancer drug}

In this issue of the JCI, Fernández et al. (13) present a novel approach using structurebased rational drug design and medicinal chemistry to reengineer imatinib in order to retain its anticancer activity, but importantly, without the risks of cardiotoxicity (Figure 1). Reengineering a drug substance, 
or "scaffold," based on structural insights is an artful process in which technology and imaginative creativity combine. The team of Fernández et al. made some fundamental choices in this work. First, they chose to focus on retaining the KIT-inhibitory activity of imatinib as their primary aim, while diminishing the ABL-inhibitory activity of the reengineered compound. The reasoning for this was because the mutated version of KIT is a valid oncogenic target in GISTs and also now so for a subset of melanomas (14). "Dialing out" the ABL-inhibitory activity would, of course, make the newly reengineered agent less useful as a treatment for CML, but the hope of these investigators was to create a less cardiotoxic drug that could be used for the treatment of GIST and other KIT-dependent malignancies. The authors used some novel chemical insights to design the new compound. By examining the de-wetting differences across two imatinib targets (KIT and ABL), they were able to use a measure of hydration tightness to determine how best to modify the structure of imatinib to diminish $\mathrm{ABL}$ binding while preserving the KIT binding. These techniques allowed the investigators to sculpt a new inhibitory ligand based on de-wetting differences that would enhance the desired activity and repress the undesirable elements of its actions. These structural modifications resulted in the new compound, which they christened WBZ_4. WBZ_4 is structurally distinct from imatinib, representing a methylated variant on the parental structure. The methyl group added to imatinib in WBZ_4 was able to preserve the inhibitory activity in KIT binding while decreasing the association of this new molecular entity with BCR-ABL. Interestingly, because earlier laboratory studies suggested that JNK inhibition may protect against cardiac myocyte damage, the authors chose to also design WBZ_4 to possess increased inhibitory activity against JNK so that cardioprotection of the new agent would be doubly fortified. WBZ_4 was then tested both in vitro and in vivo, to prove both that the selective anticancer activity of this new molecule was maintained in GIST cell lines as well as a mouse model of GIST and that cardiotoxicity was reduced in the animals exposed to WBZ_4 compared with those exposed to imatinib.

\section{Challenges for a reengineered new drug}

As with any drug design effort, some predefinition of target validity was necessary to serve as the foundation for this work. In the present study (13), tyrosine kinases $\mathrm{ABL}$ and JNK were identified as targets for which less and more inhibition, respectively, would be desirable in a newly engineered compound. These are certainly reasonable assertions, but human physiology tends to be complex, filled with multiple levels of redundant and intersecting intracellular signaling pathways. It is of interest that a major activity of imatinib, the inhibition of PDGFRs, is not commented upon by the authors as a target to optimize or to minimize in the profile of the reengineered agent WBZ_4. Inhibition of the tyrosine kinase activity in the PDGFR family has been well documented to induce changes in intratumoral interstitial pressures and change the fluid homeostasis among the tumor compartment, vascular compartment, and extracellular space (15). This could be one very important reason why patients who receive PDGFR inhibitors, including imatinib, develop edema and third-spacing of fluid (mobilization of fluid to a location within the body rendering it unavailable to the circulatory system). Thus, inhibition of PDGFR signaling, by contributing to edema and volume increase in patients, could stress the cardiovascular system of a patient and induce cardiotoxicity through indirectly putting extra strain on the heart Because the cardiovascular impact of PDGFR signaling blockade remains somewhat unclear, it would be important to assess in advance whether WBZ_4 has the same PDGFR inhibition as the parental drug, imatinib.

It is also notable that small-molecule drugs will never be completely specific for any single physiologic or pathophysiologic pathway. Optimizing one element of an agent may unpredictably change some other property of the compound. In this case, the desirable pharmacologic properties of imatinib may be impacted negatively by the molecular modifications made in this study (13), potentially rendering the WBZ_4 molecule less bioavailable. Other activities might also appear with more broad-based molecular profiling: small structural changes to small-molecule drugs may change the spectrum of targets to which that drug binds.

\section{Summary}

One of the most important pharmacologic insights of the past few decades has been the degree to which selectivity can be engineered into kinase-inhibiting drugs.
Although most small-molecule tyrosine kinase inhibitors bind to the ATP-binding site of target kinases, there are sufficiently distinct variations between these ATPbinding pockets to confer a reasonable amount of selectivity onto novel smallmolecule chemical entities. This allows new drugs to be fashioned rationally based on structural understanding of the ligand-binding interactions as well as on a deep understanding of the biology of the target enzyme. The approach used here by Fernández et al. (13) holds great promise to allow more customized development of rationally designed therapeutic agents. The structure-based construction of potential drug-like molecules with various "flavors" of action, from simple and selective to complex and multiple, will present translational and clinical investigators with many tools to dissect the pathways that drive human disease. It will also challenge the capacity of our clinical development systems, because we will need more predictive tests to define which agents are likely to improve substantively the impact of currently available agents that have, on the whole, impressively tolerable safety profiles for the degree of clinical benefit that they offer patients with these lifethreatening malignancies. The first generation of kinase-inhibitory drugs such as imatinib and sunitinib have already provided patients with life-saving therapeutic options, and with tools such as those described by Fernández et al., the future certainly looks bright for constructing ever-better agents that can be combined safely and effectively to manage, and eventually cure, many forms of human cancer.

Address correspondence to: George D. Demetri, Ludwig Center, Dana-Farber Cancer Institute, Dana 1212, 44 Binney Street, Boston, Massachusetts 02115, USA. Phone: (617) 632-3985; Fax: (617) 632-3408; E-mail: gdemetri@partners.org.

1. Levitzki, A. 2002. Tyrosine kinases as targets for cancer therapy. Eur. J. Cancer. 38(Suppl. 5):S11-S18.

2. Capdeville, R., Buchdunger, E., Zimmermann, J., and Matter, A. 2002. Glivec (STI571, imatinib), a rationally developed, targeted anticancer drug. Nat. Rev. Drug Discov. 1:493-502.

3. Druker, B.J., et al. 2006. Five-year follow-up of patients receiving imatinib for chronic myeloid leukemia. N. Engl. J. Med. 355:2408-2417.

4. Demetri, G.D., et al. 2002. Efficacy and safety of imatinib mesylate in advanced gastrointestinal stromal tumors. N. Engl. J. Med. 347:472-480.

5. Verweij, J., et al. 2004. Progression-free survival in gastrointestinal stromal tumours with high-dose imatinib: randomised trial. Lancet. 364:1127-1134.

6. Demetri, G.D., et al. Efficacy and safety of sunitinib 
in patients with advanced gastrointestinal stromal tumour after failure of imatinib: a randomised controlled trial. Lancet. 368:1329-1338.

7. Desai, J., et al. 2007. Clonal evolution of resistance to imatinib in patients with metastatic gastrointestinal stromal tumors. Clin. Cancer Res. 13:5398-5405.

8. Talpaz, M., et al. 2006. Dasatinib in imatinib-resistant Philadelphia chromosome-positive leukemias. N. Engl. J. Med. 354:2531-2541.

9. Kerkela, R., et al. 2006. Cardiotoxicity of the cancer therapeutic agent imatinib mesylate. Nat. Med. 12:908-916
10. Force, T., Krause, D.S., and Van Etten, R.A. 2007. Molecular mechanisms of cardiotoxicity of tyrosine kinase inhibition. Nat. Rev. Cancer. 7:332-344.

11. Verweij, J., et al. 2007. Imatinib does not induce cardiac left ventricular failure in gastrointestinal stromal tumours patients: Analysis of EORTC-ISGAGITG study 62005. Eur. J. Cancer. 43:974-978.

12. Khakoo, A.Y., et al. 2007. Rare incidence of congestive heart failure (CHF) in gastrointestinal stromal tumor (GIST) and other sarcoma patients (pts) receiving imatinib mesylate (IM) therapy. In ASCO Annual Meeting Proceedings Part I. Vol.
25, no. $18 \mathrm{~S}$ (June 20 supplement). J. Clin. Oncol. 2007:10026.

13. Fernández, A., et al. 2007. An anticancer CKit kinase inhibitor is reengineered to make it more active and less cardiotoxic. J. Clin. Invest. 117:4044-4054.

14. Hodi, F.S., et al. 2007. Major response to imatinib mesylate in KIT mutated melanoma. J. Clin. Oncol. In press.

15. Ostman, A., and Heldin, C.H. 2007. PDGF receptors as targets in tumor treatment. Adv. Cancer Res. 97:247-274.

\title{
The weight of cell identity
}

\author{
David T. Scadden
}

Center for Regenerative Medicine, Massachusetts General Hospital, Boston, Massachusetts, USA. Harvard Stem Cell Institute and Department of Stem Cell and Regenerative Biology, Harvard University, Cambridge, Massachusetts, USA.

\begin{abstract}
Recent studies involving molecular modification of adult somatic cells have pointed to a remarkable plasticity in cell identity. In this issue of the JCI, Koh and colleagues assessed whether bone marrow-derived cells could alter their fate under circumstances conducive to adipocyte generation in vivo (see the related article beginning on page 3684). These cells remained true to their roots, indicating how difficult it will be to exploit cell plasticity for therapeutic purposes.
\end{abstract}

Fat, the ever-popular source of personal and now societal anxiety, is of great scientific interest as a multidimensional regulator of health and longevity. Its origins have, like many cells of mesenchymal lineage, been explored to only limited extent. Progenitor cells of adipose tissue, bone, and cartilage are thought to descend from mesodermally derived multipotential mesenchymal stem cells (MSCs). The presence of MSCs in adult tissues has been demonstrated in mice and humans, but how they contribute to the maintenance or repair of adult tissues has not been defined, in part due to the lack of MSC-specific promoters that would enable lineage-tracking experiments. Where MSCs reside within tissues was also obscure; therefore, tracking these cells by in situ labeling was also difficult. Now it appears they reside in a perivascular location (1). Similarly, the developmental origin of MSCs has not been well characterized and is presumed to be mesodermal, but new information indicates that some MSCs derive from the neural crest (2).

Nonstandard abbreviations used: iPC, induced pluripotent cell; MSC, mesenchymal stem cell.

Conflict of interest: The author is a stockholder in Fate Therapeutics Inc.

Citation for this article: J. Clin. Invest. 117:3653-3655 (2007). doi:10.1172/JCI34181.

\section{Adipogenesis and adipocyte progenitor cells}

Adipocytes of both brown and white fat are thought to descend from MSCs by poorly defined intermediates and can respond in activity and number to a range of different stimuli. Adipocytes can, perhaps unfortunately, regenerate in settings of destruction and do have the capacity to transition between brown and white adipocytes. Whether they increase in number by proliferation as mature cells, amplification of precursor cell populations, or development from MSCs is not clear. Recently, it was reported by Crossno et al. that, in mice, adipocytes may derive from blood-borne cells under specific conditions: a high-fat diet or treatment with the antidiabetic drug rosiglitazone (3). In this issue of the JCI, that conclusion is drawn into question by studies of similar design reported by Koh et al. (4). In both studies $(3,4)$ bone marrow containing a genetic tracer (GFP) was transplanted from a donor mouse into a recipient mouse. The contribution of the donor bone marrow-derived cells to the adipocyte population was then assessed in the recipient animals. The analytical methods used in the two studies were different. Greater singlecell precision via the use of microscopy was achieved in the Koh et al. study, suggesting that evidence of bone marrow-derived cell contributions to adipocytes reported by Crossno et al. (3) was perhaps the result of inadequate discrimination of adipocytes from neighboring macrophages. In contrast, Crossno et al. examined GFP-positive adipocytes by flow cytometry and could detect no hematopoietic markers (CD45 and CD11b) in GFP-positive adipocytes. Since macrophages in fat do phagocytose adipocytes and become multinucleated, it may be that the fused cells have lost detectable CD45 or CD11b and yet continue to have detectable levels of GFP. With the new data reported in this issue, it is increasingly difficult to accept the alternatives that bone marrow-derived mesenchymal precursors form distant adipocytes or that adipocytes have the ability to be generated by hematopoietic cell populations.

\section{Fate specification and plasticity}

The notion that cells might take on an alternative fate even after the specification of identity has been an intriguing concept for some time, with waxing and waning evidence inspiring debate. A change in cellular fate might occur through two basic processes: conversion from one mature cell type to another, called transdifferentiation; or reversion to a less-differentiated state with subsequent maturation along alternative cell lineages, called dedifferentiation. Each of these processes would involve a reprogramming process that presupposes a great deal of plasticity in the mature cell.

That these processes can occur experimentally was defined decades ago by John Gurdon's work transferring the nucleus of a Xenopus enterocyte into an enucleated Xenopus oocyte (5). In that setting, he 